\title{
Disaster Detection Using SVDD Group Learning for Emergency Rescue Evacuation Support System
}

\author{
Tomotaka Wada ${ }^{1 *}$, Hiroko Higuchi ${ }^{1}$, Ken Komaki ${ }^{1}$, Haruka Iwahashi ${ }^{1}$, and Kazuhiro \\ Ohtsuki $^{2}$ \\ ${ }^{1}$ Department of Engineering Science, Kansai University \\ ${ }^{2}$ Graduate School of Intercultural Studies, Kobe University \\ *t-wada@ieee.org
}

Received: August 19, 2015; Accepted: January 13, 2016 ; Published: August 8, 2016

\begin{abstract}
Many people have got injured and died by sudden disasters such as fires and terrorisms. We have proposed an Emergency Rescue Support System (ERESS) for reducing victims at the time of disaster. ERESS operates under mobile ad-hoc networks (MANET) composed of handheld terminals such as smartphones and tablets. ERESS terminals have disaster detection algorithm and plural sensors such as acceleration, angular velocity, and geomagnetism. ERESS detects the disaster from the behavior analysis of people by the sensors. In this paper, we propose a new disaster detection method by performing the machine learning in the group using a support vector domain description (SVDD). We are able to detect the abnormal behavior of people by using this method. The results of disaster simulation experiments show the validity of the proposed method.
\end{abstract}

Keywords: ERESS, MANET, evacuation support system, group learning, SVDD

\section{Introduction}

Many people have got injured and died by sudden disasters such as fires, earthquakes and terrorisms. People under these disasters cannot make a cool judgment by falling into the panic state. The people of the panic state may take unexpected actions. Hence, these actions may cause a second disaster and lead to expansion of the damage. For the reduction of human damage by the disaster, it is important how quick you can start evacuation in the limited time just after the outbreak of disasters.

As one of the evacuation support systems that are able to detect disasters early and give evacuate guidance, there is a sensor network $[1,2]$. This system needs to install various sen- 


\section{Journal of Advanced Simulation in Science and Engineering}

sors (e.g. gas, smoke, heat, sound, etc.) and servers in the building. If abnormality is detected in the building by managing the information obtained from sensors with servers, it sounds a warning. However, this system has serious problems as follows.

(1) No working under destroyed machines:

When sensors or servers are destroyed by disasters, the system is not able to work. In addition, when the communication networks are destroyed, the system is not able to collect information of disaster. So the whole system is not able to work properly.

(2) Only sensing environmental factor:

It is superior in the detection of disasters by the environmental changes such as fires. But, it cannot sense the state of people in the disaster. Moreover, it cannot cope with disasters caused by the change of action of people such as terrorism.

(3) High cost:

It is necessary to install various sensors and servers in a building. Since there are a lot of sensors and servers, the system becomes expensive. In addition, it needs costs for its maintenance.

On the other hand, there is a disaster navigation system as other evacuation support systems [3]. This system can identify the direction and the distance from the present location to the nearest evacuation center or home by using a cellular phone. However, this system has an important problem that it cannot know evacuees in real time. In addition, when infrastructure to offer information is destroyed, evacuees cannot use this system.

We have proposed an evacuation support system named Emergency Rescue Evacuation Support System (ERESS) which can work without depending on neighboring infrastructure to solve the above problem [4-9]. The purpose of ERESS is to significantly reduce the number of victims by starting evacuation guidance within 30 seconds just after the outbreak of the sudden disaster. ERESS works by using mobile terminals called ERESS terminals and has following three characteristics.

(1) Operable in various environments:

ERESS does not need infrastructure to communicate between mobile terminals using MANET. If there are multiple terminals in the neighboring, they are able to configure mesh networks. So it can work anywhere not depending on infrastructure.

(2) Automatic detection of disasters:

ERESS analyzes the behavior of terminal holders using data from sensors mounted on ERESS terminals. The system detects a disaster from the movement of people in real time by using the analysis of ERESS terminals.

(3) Low cost:

ERESS works by only using smartphones or tablet terminals spreading widely. Hence a dedicated high cost equipment for a building is not necessary. So we are able to receive evacuation support in any places. 
The purpose of this paper is accuracy improvement of the automatic detection of the disaster in ERESS. In the conventional ERESS, it is necessary for us to learn behavior by making training data beforehand for the disaster detection. However, it is not realistic to learn each behavior because it is annoying for us. So we propose a new effective disaster detection method using a support vector domain description (SVDD). Learning from the behavior of everyday people becomes possible by using SVDD. We are able to improve accuracy of disaster detection by detecting the difference between the everyday behavior.

This paper is composed as follows. In section 2, we describe ERESS. In section 3, we propose a new disasters detection method using a SVDD. In section 4, we choose sensor axis to use for the proposed method by fundamental experiment. In section 5 , we show performance evaluations by experiments. Finally, we conclude this paper in section 6 .

\section{ERESS}

In this section, we explain the basic constitution of ERESS. The purpose of ERESS is to reduce victims drastically by starting evacuation support within 30 seconds just after the outbreak of a sudden disaster. ERESS detects the sudden disaster using mobile terminals, offers emergency evacuation information to evacuee in real time. This would decrease the number of victims. The system is composed of five phases. We show the function of the system. Fig. 1 shows a concept of ERESS.

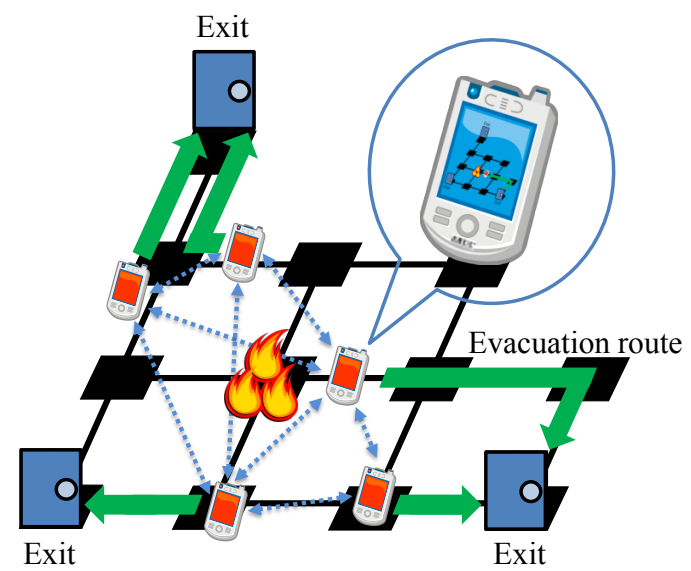

Figure 1: Concept of ERESS

\subsection{Exchanging and sharing information by MANET (Phase 1)}

ERESS uses MANET as a communication method. This method builds a dynamic network only using mobile terminals. Hence, the construction of the network is possible at every place without depending on the infrastructure. ERESS terminals provide the emergency evacuate support information to evacuees in real time by exchanging and sharing information with 
neighboring terminals at the time of disaster. We are developing it by computer simulations [4]. The information includes the following three contexts.

(1) Occurrence location of disasters and dangerous area

When a fire or terrorism occurs, dangerous areas spread with time. Terminal holders have to avoid dangerous areas by knowing the occurrence location of the disaster from ERESS terminals and can evacuate safely.

\section{(2) Crowded route}

When disasters occur, evacuees go to exits to go away from dangerous areas. At this time, passages leading to exits are crowded, and evacuation becomes difficult. Because the terminals always communicate with others, terminals display the evacuation route which avoids places that the density of terminals is high.

(3) Information about life and death

When the terminal holder does not move after disasters occurred, the person is likely to have fallen into a dangerous situation. In this case, the terminal sends the emergency information to others, and helps to call a rescue team.

\subsection{Behavior analysis of terminal holders (Phase 2)}

ERESS terminals have multiple sensors and always sense terminal holder's behavior, and analyze the behavior state from the sensing data. The behavior analysis also affects the disaster detection. Therefore this phase is very important. We are developing it by Pre-ERESS [5]. The kind of sensor used in this phase is classified into three factors.

(1) Physical factors: The information to get directly from the behavior of terminal holders. (e.g. acceleration, vibration, and direction)

(2) Psychological factors: The biological information about psychological situation of terminal holders. (e.g. heat rate, blood pressure, and sweating)

(3) Environmental factors: The information that shows neighboring environment. This includes terminals density indicating the density of evacuees. (e.g. smoke, temperature, and GPS)

\subsection{Disaster detection (Phase 3)}

The disaster detection algorithm in ERESS uses SVM (Support Vector Machine) and simple statistics information. SVM is one kind of the machine learning, the classification of two classes is possible [6-8]. ERESS terminals identify emergency state by using the SVM for the data obtained in Phase 2, and collect the information of neighboring terminals by MANET. When the number of terminals of the emergency state exceeds a threshold, ERESS will detect 
disaster. The terminals which detected disaster transmit information of disaster outbreak to other terminals and encourage the evacuation of other terminal holders. We know that the accumulation of the emergency state can be approximated to the logistic curve. We may identify the kind of the disaster by analyzing the incline of this curve.

\subsection{Evacuation route search (Phase 4)}

This phase is evacuation route search. It is necessary for evacuees to evacuate safely and quickly when the disaster occurs. When a fire occurs, the fire spreads through neighbors and grows bigger. In the case of terrorism, terrorists may move. So, because dangerous areas change with time, it is necessary to respond to changes in environments. Then first, ERESS calculates path costs in consideration of dangerous areas of neighborhood of disaster outbreak position and the congestion situation of passages. Second, we use Dijkstra algorithm [9]. This algorithm is a method to decide the route where evacuees can evacuate to most safely and quickly at lowest cost.

\subsection{Evacuation route display and guidance (Phase 5)}

When the search of the evacuation route is finished, ERESS terminals display the present location and an appropriate evacuation route and disaster outbreak point on the own screen and it performs the evacuation using the maps and voice. Terminal holders can evacuate safely and quickly by following routes that ERESS terminals display.

ERESS is not enough now. The system has problems. First, in conventional method, we classify the behavior (stop, walk, and run) of terminal holders by using SVM. To perform the behavior classification, it is necessary to create the training data. The training data is required answer pair of data. For example, we must prepare the correspondence of the data about all behaviors. It is necessary for terminal holders to do the decided behavior to make data group of each behavior. However, it is not realistic for the conventional method to apply to actual environments. In addition, as for the conventional system, since the number of the classifications is three of stop, walk, and run, we regard the run as a dangerous behavior related with disasters. However, dangerous behaviors also can be applicable to sudden stop, sudden acceleration and falling down. When we increase the number of the classifications to classify all the dangerous behavior, classification accuracy might be decrease. In addition, it is necessary for terminal holders to make the data to let terminals learn dangerous behavior. Second, the conventional disaster detection method often makes false detection. As for the conventional method, it detects a disaster if the number of running state exceeds the majority of the total number of terminals. However, this method causes false detection in several cases.. For example, there is rushing to the train or crossing at the crosswalk. The false detection might lead to a drop of the reliability of the system.

\section{Proposed method}

In this section, we consider a method to solve problems that the current ERESS has. A struc- 


\section{Journal of Advanced Simulation in Science and Engineering}

ture to detect a disaster correctly is necessary to solve the problem. We consider to judge dangerous behaviors from data of daily behaviors. Therefore, we propose a disaster detection method based on group learning using SVDD.

\subsection{Purpose and characteristic}

There are two purposes of the proposed method. First, it improves the detection precision of disasters by classifying the emergency state from the data in the normal state of daily life. Second, it builds a system suitable for actual environments on daily life.

The characteristic of the proposed method is that the learning of behavior in a normal state is possible only from every data by using SVDD used for abnormality detection. Hence, we can analyze the abnormal behavior of terminal holders at the time of disaster outbreak. In addition, it performs disaster detection from the state of neighboring terminals by performing group learning using the state of other terminal holders. The data for use is acceleration and vibration that are mounted on mobile terminals.

\subsection{Support Vector Domain Description (SVDD)}

SVDD is unsupervised learning [10]. Unsupervised learning is to perform learning from no training data of the class label. We estimate a high density domain by considering the whole of no training data of the class label to be one class. SVDD estimates a high density domain as a sphere that is shown in Fig. 2. In other words it calculates the sphere that the spherical volume including all of the training patterns is minimized. Support vectors are selected from training patterns to construct the minimum circle which includes all training patterns. For unknown input, we distinguish the spherical inside or outside, and classify normal state and emergency state. In this paper, we use acceleration and vibration of the daily life as training data. From the smallest sphere including those data, we judge the state of terminal holders at the time of disasters.

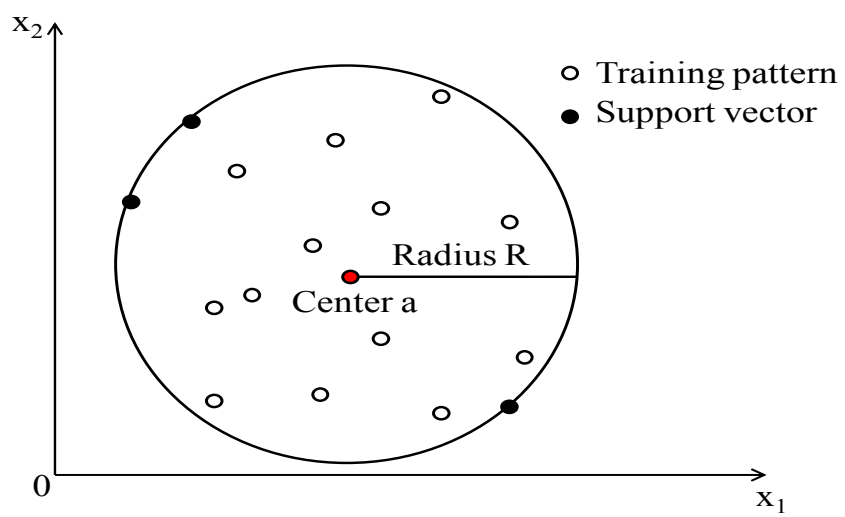

Figure 2: Training pattern and support vector in SVDD 


\section{Journal of Advanced Simulation in Science and Engineering}

\subsection{Proposed algorithm}

The proposed algorithm is classified roughly into two of a learning stage and judgment stage. In the learning stage, each ERESS terminal learns the normal state based on daily data. In the judgment stage, it judges disaster or not. Fig. 3 shows summary of the proposed algorithm.

(1) Learning stage

[Step 1: Calculate personal data]

ERESS terminals calculate average and variance of acceleration and vibration during $\mathrm{T}$ seconds as personal data.

[Step 2: Calculate deviation from personal data]

ERESS terminals calculate deviation from normalized personal data to share data with other terminals.

[Step 3: Neighboring terminal's state collection

ERESS terminals collect the deviation data of other terminals to perform group learning.

[Step 4: Calculate learning circle $\alpha$ using SVDD]

The learning circle $\alpha$ is constructed in central coordinate and radius R. R calculated on the learning stage becomes the threshold to use for disaster judgment stage.

(2) Judgment stage

[Step 1: Calculate the deviation of each terminal holder]

ERESS terminals calculate deviation during $\mathrm{M}$ seconds which is shorter than $\mathrm{T}$ seconds used in the learning stage. We can catch the change of neighboring terminal's state quickly.

[Step 2: Neighboring terminal's state collection]

As with the learning stage, ERESS terminals collect the deviation data of other terminals to perform group learning.

[Step 3: Calculate judgment circle $\beta$ using SVDD]

The judgment circle $\beta$ is constructed in central coordinate and radius $r$. The value of $r$ is determined by the emergency state terminal's data.

[Step 4: Disaster judgment using SVDD value]

In the disaster judgment, we use the SVDD value. The value is calculated from radius $r$ of the judgment circle $\beta$ and a ratio of the number of the data located outside of the learning circle is e. When the total number of data is $n$, and the total number of data on the outside of the learning circle is e, SVDD value is shown in the following equation.

$$
\text { SVDD value }=\mathrm{r} \times \mathrm{e} / \mathrm{n}
$$

Disaster Judgment: $\mathrm{R}<\mathrm{SVDD}$ value

Normal Judgment: $\mathrm{R}>$ SVDD value

By using the SVDD value, we can consider a ratio of terminals of the emergency state. By comparing the $\mathrm{R}$ with the SVDD value, disaster judgment is performed. Hence, accurate disaster occurrence detection is possible.

We show the system flow of the proposed method in Fig. 4. The group learning is equivalent to the learning stage in the proposed method procedure. We update the value of the threshold to use for disaster judgment whenever we perform the judgment stage at $N^{\prime}$ times. After N' times, we go back to the group learning in learning stage. This process is repeated. So the whole algorithm of the proposed method is (b) judgment stage including group learning of (a) learning stage. 


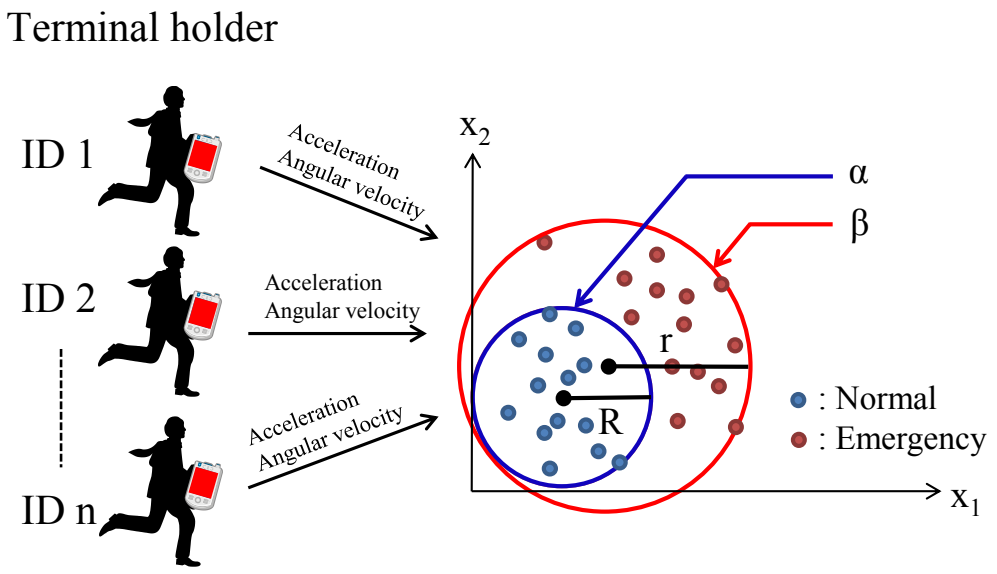

Figure 3: Proposed method

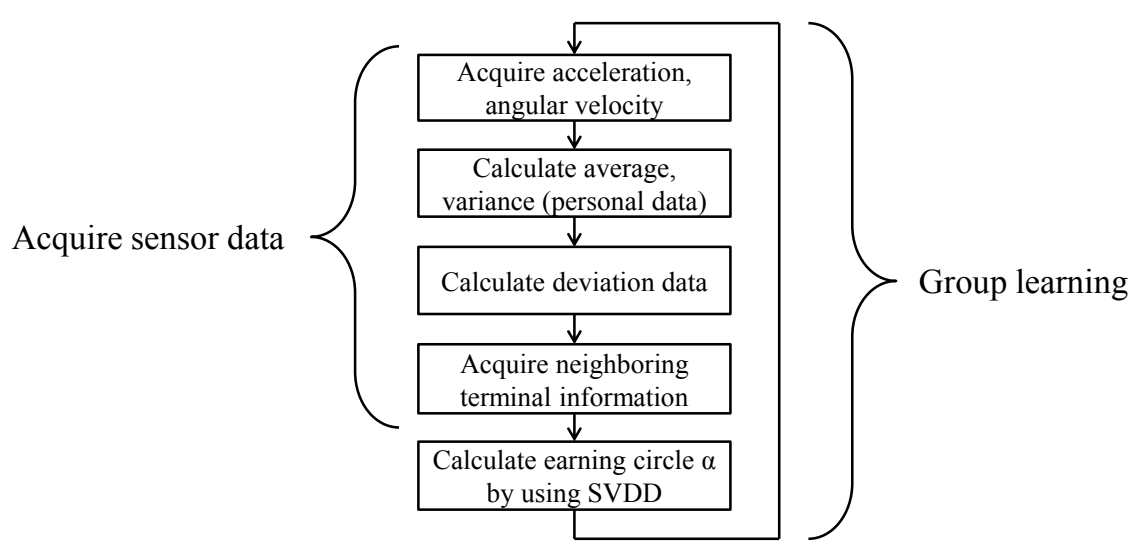

(a) Learning stage

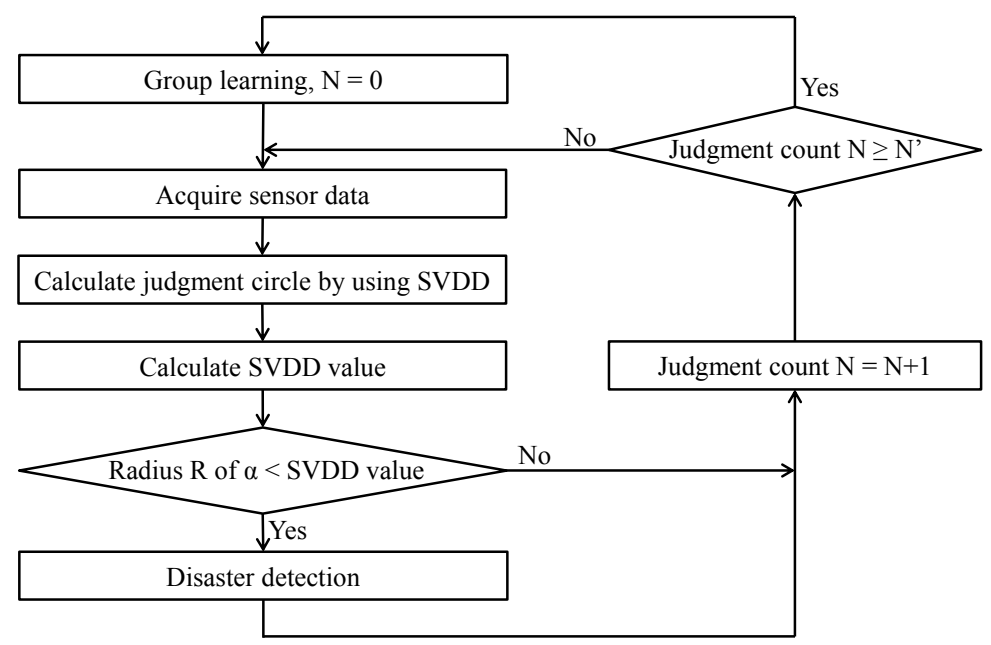

(b) Judgment stage

Figure 4: System flow of the proposed algorithm 


\section{Fundamental experiments}

In this section, we choose the axis of the sensors to use in the proposed algorithm. The proposed algorithm uses an acceleration sensor and an angular velocity sensor to acquire the detailed state of terminal holders. Each sensor has 3 axes of $\mathrm{x}, \mathrm{y}$ and $\mathrm{z}$. When we acquire data for all axes, the amount of the data becomes enormous. So it needs more time to process the data. Hence it is necessary for us to choose the axis of the sensors to use by the proposed algorithm.

\subsection{Summary of experiments}

We acquire the data of the examinees to consider the axis of sensors in the fundamental experiments for disaster simulation. The experimental conditions are shown in Table 1. Figs. 5 and 6 show the experimental ERESS terminal and three axes of the sensor, respectively. We attach sensors to the waist. These experiments are carried out by assuming terrorism. The experimental method is that a terrorist who has a knife invades a room suddenly and takes action to slash at people, and then the examinees leave the room while escaping from the terrorist. We compare 3 patterns of angular velocity in $\mathrm{x}$-axis, $\mathrm{y}$-axis and z-axis. We use the value of acceleration in $\mathrm{z}$-axis. This is because the $z$-axis of the acceleration has the most characteristic in our conventional study.

Table 1: Experimental conditions

\begin{tabular}{|c|c|}
\hline Size of experimental field & $19.6[\mathrm{~m}]^{*} 15.8[\mathrm{~m}]$ \\
\hline The number of examinees & $14[$ person $]$ \\
\hline Creation time of learning circle & $40[\mathrm{~s}]$ \\
\hline Creation interval of judgment circle & $1[\mathrm{~s}]$ \\
\hline Disasters type & Terrorism \\
\hline
\end{tabular}

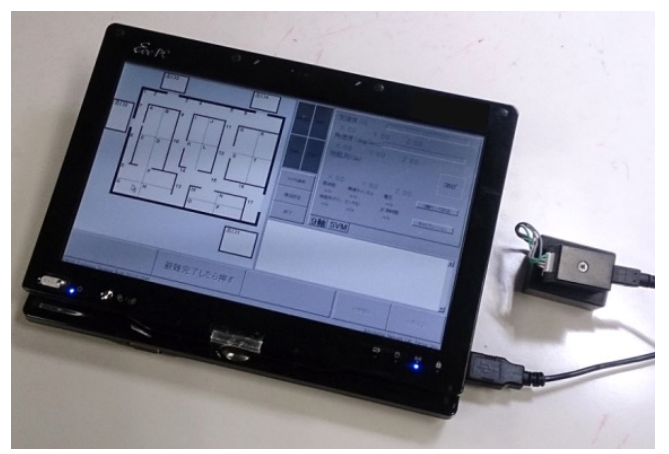

Figure 5: ERESS terminal and 9 axes sensor 


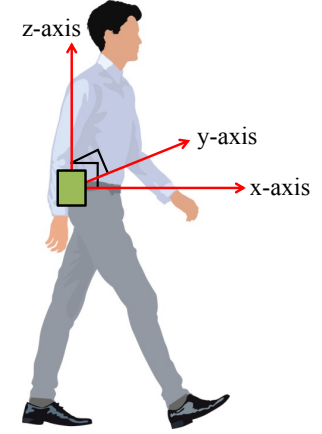

Figure 6: Sensor axes

\subsection{Experimental results}

Fig. 7 shows experimental results using $\mathrm{x}$-axis of the angular velocity. The horizontal axis is time, and the vertical axis is the SVDD value decided in eq. (1). As a result, we were able to detect terrorism definitely when the SVDD value was exceeded the threshold at approximately 12 seconds after the terrorism outbreak. The threshold is determined by radius $\mathrm{R}$ of learning circle in learning stage. However when we used $y$-axis or z-axis of angular velocity, we couldn't detect terrorism since SVDD value was not exceeded the threshold. Hence, we use acceleration in z-axis and angular velocity in $\mathrm{x}$-axis of sensors to use in the proposed algorithm.

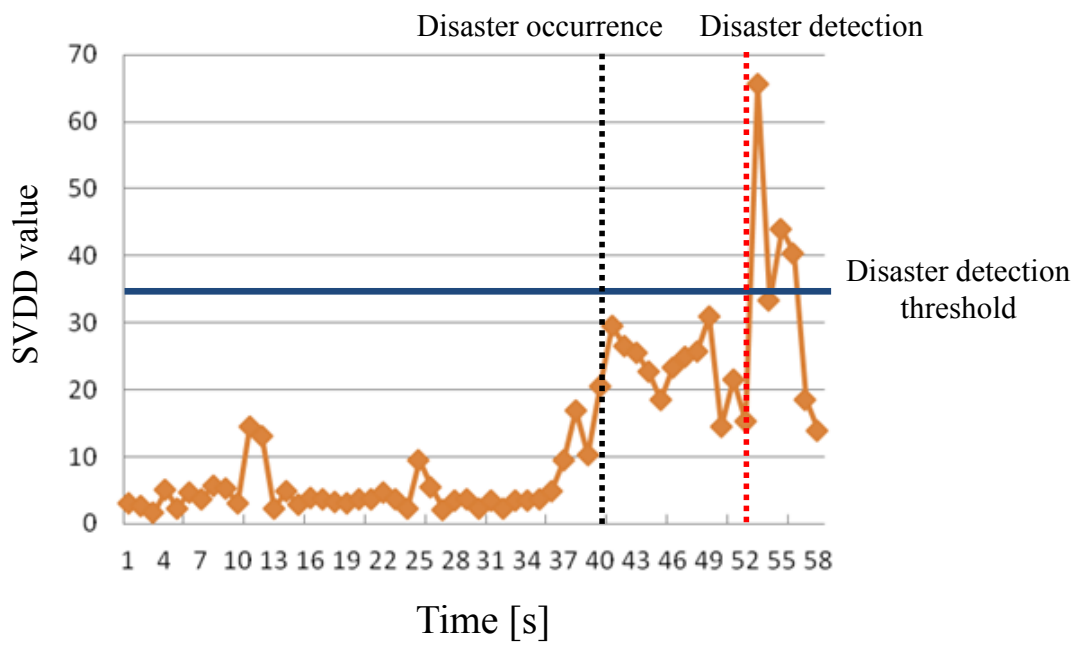

Figure 7: SVDD value

\section{Performance evaluations by experiments}

In this section, we conduct disaster simulation experiments and evaluate the performance of 


\section{Journal of Advanced Simulation in Science and Engineering}

disaster detection. The purpose of the experiments is to compare the proposed method with the conventional method about the disaster detection rate and disaster detection time that are important in ERESS. The conventional method is the method that detects disasters if the majority of the total number of terminals become emergency states. We acquire the behavior of evacuees who faced disasters in the following two kinds of experiments. In order to assume disaster occurrence during a lecture, experiments are conducted in a class room of our university. Table 2 and Fig. 8 show the experimental conditions and the experimental field, respectively.

Table 2: Experimental conditions

\begin{tabular}{|c|c|}
\hline Size of experimental field & $40[\mathrm{~m}] * 50[\mathrm{~m}]$ \\
\hline The number of examinees & $87[$ person $]$ \\
\hline A average number of examinees per experiment & $30[$ person / experiment $]$ \\
\hline Creation time of learning circle & $40[\mathrm{~s}]$ \\
\hline Creation interval of judgment circle & $1[\mathrm{~s}]$ \\
\hline Experiments type & Evacuation in different emergency \\
& Terrorism \\
\hline
\end{tabular}

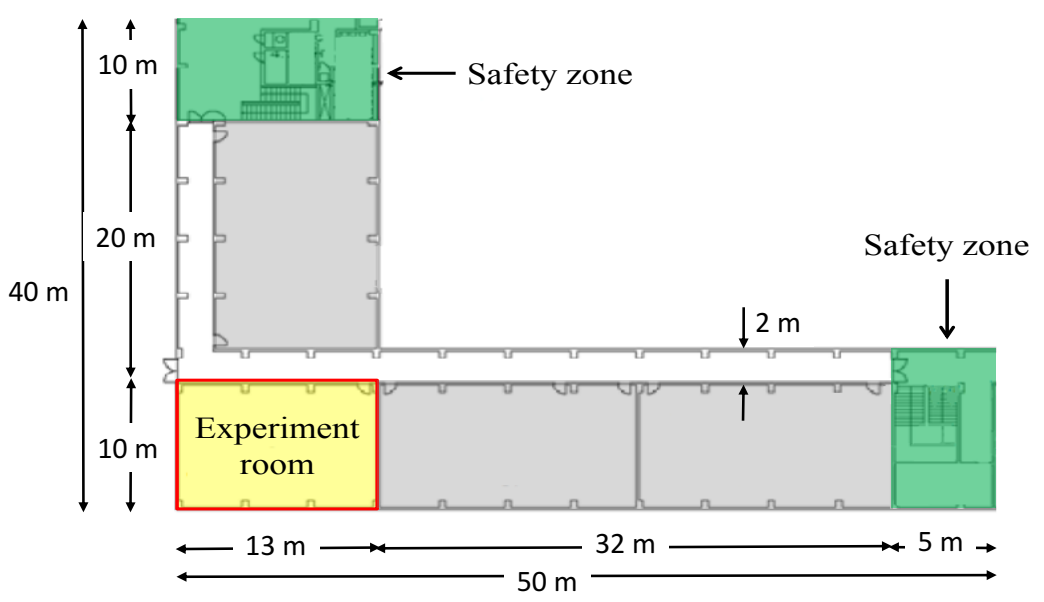

Figure 8: Experimental field

\subsection{Summary}

(1) Evacuation experiments in different urgency

We performed two types of experiments in normal and emergency states which are different urgency of evacuation. Examinees located in the experiment room evacuate to the safe zone in the emergency. On the other hand, people run quickly to ride on a train with hurry in daily 


\section{Journal of Advanced Simulation in Science and Engineering}

life. Such the situation is assumed to be a low-urgent degree. In experiments of the emergency state, all examinees have to evacuate to the safe zone as rapidly as possible. Such the situation is assumed to be a high-urgent degree. We compare the disaster detection rate of the conventional and the proposed method in two types of experiments.

\section{(2) Experiments of terrorism}

In these experiments, a terrorist who has a gun breaks into the experiment room and attacks examinees. They are not informed what happens from now on. So, we can acquire the behavior of examinees encountered in terrorism in the environment close to the actual situation.

\subsection{Experimental results}

In order to compare the conventional method and the proposed method, we use the daily data such as each 10 seconds of stopping, walking, running, and stopping. Fig. 9 shows the learning circle obtained by SVDD. We normalize the data by average and variance of 40 seconds data. One plot is the normalized value during 0.1 second in Fig. 9. Since the values of acceleration and angular velocity are the normalized values, the values have no unit. The lerning is carried out by data of five people. When the radius $\mathrm{R}$ of learning circle is large, the area of normal state is large. So the possibility of disaster detection becomes small. On the other hand, the possibility of disaster detection becomes large since the area of normal state is small in small $\mathrm{R}$.

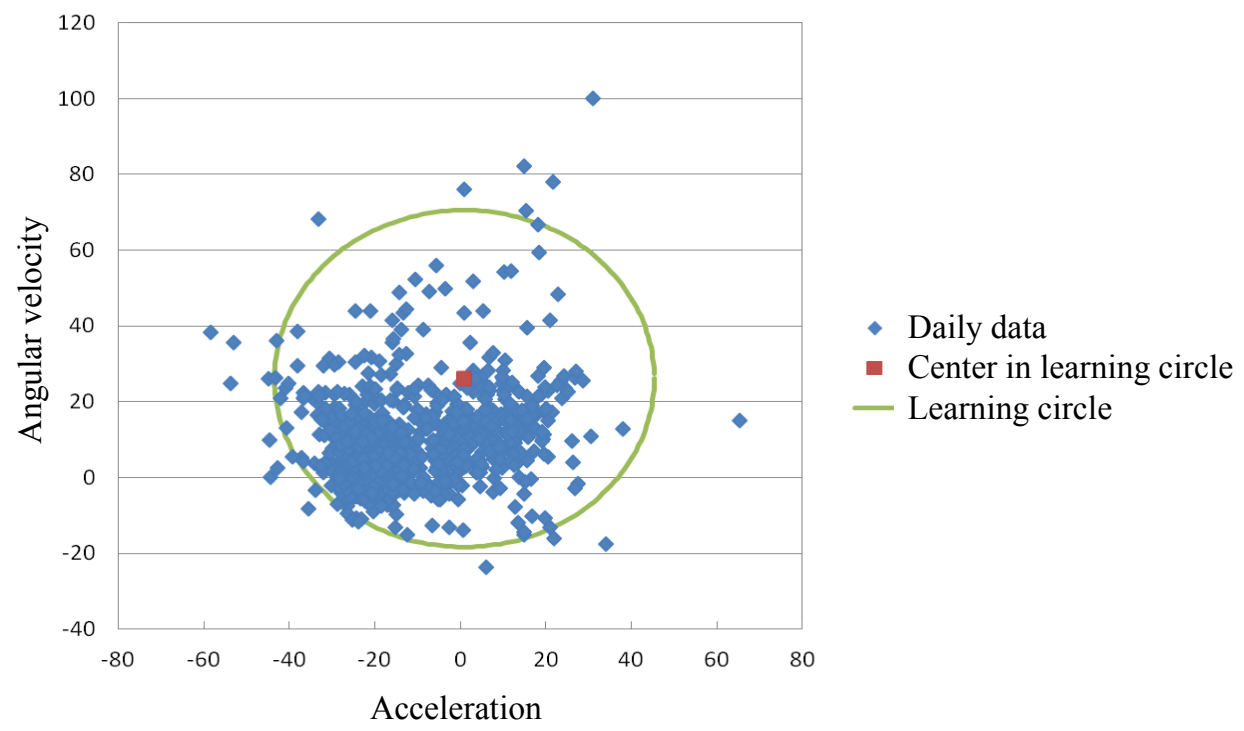

Figure 9: Learning circle obtained by SVDD 


\section{Journal of Advanced Simulation in Science and Engineering}

(1) Evacuation experiments in different urgency

Table 3 shows results of the conventional and proposed methods in the different urgency. In this table, when experiments of low-urgent degree are judged to be disasters, it is written as erroneous detection. If it is judged the normal state, it is written as non-detection. When experiments of high- urgent degree are judged to be disasters, it is written as detection. The case that disasters are detected only in the experiments of high-urgent degree was written as success. In low-urgent degree, we assumed the situation that many people run in daily life such as dashing onto a train in hurry. The conventional method caused the erroneous detections in all experiments. In contrast, the proposed method was able to reduce the erroneous detections. In the high-urgent degree, each method was able to detect all experiments. As a result, the proposed method is able to judge the difference in urgent degree, and the disaster detection rate in these experiments shows $71 \%$. So the proposed method is able to improve the judgment rate in comparison with the conventional method.

Table 3: Judgment rate

\begin{tabular}{|c|c|c|c|c|c|c|c|c|c|}
\hline & \multicolumn{7}{|c|}{ Number of times } & \multirow{2}{*}{$\begin{array}{c}\text { Judgment } \\
\text { rate[\%] }\end{array}$} \\
\hline & & 1 & 2 & 3 & 4 & 5 & 6 & 7 & \\
\hline \multirow{3}{*}{$\begin{array}{l}\text { Conventional } \\
\text { method }\end{array}$} & $\begin{array}{c}\text { Low } \\
\text { urgency }\end{array}$ & $x$ & $x$ & $x$ & $x$ & $x$ & $x$ & $x$ & \multirow{3}{*}{0} \\
\hline & $\begin{array}{c}\text { High } \\
\text { urgency }\end{array}$ & 0 & 0 & 0 & 0 & 0 & 0 & 0 & \\
\hline & Results & $x$ & $x$ & $x$ & $x$ & $x$ & $x$ & $x$ & \\
\hline \multirow{3}{*}{$\begin{array}{c}\text { Proposed } \\
\text { method }\end{array}$} & $\begin{array}{c}\text { Low } \\
\text { urgency }\end{array}$ & $x$ & $x$ & - & - & - & - & - & \multirow{3}{*}{71} \\
\hline & $\begin{array}{c}\text { High } \\
\text { urgency }\end{array}$ & 0 & 0 & 0 & 0 & 0 & 0 & 0 & \\
\hline & Results & $x$ & $x$ & 0 & 0 & 0 & 0 & 0 & \\
\hline
\end{tabular}

(2) Experiments of terrorism

Figs. 10 and 11 show examples of judgment circle in two experiments. We find the judgment circle is larger than the learning circle. Table 4 shows the detection time and the disaster detection rate of each method in experiments of terrorism. Fig. 12 shows the 4th experimental results as an example which was able to detect disaster in the proposed method. The horizontal axis is the experiment elapsed time, and the vertical axes are accumulation of the number of terminals of the emergency state and SVDD value. The threshold values of conventional and proposed methods are more than half of all terminals and radius $\mathrm{R}$ of learning circle, respectively. Disaster outbreak time is 0 second. In this experiment, the average detection time of the proposed method is only two seconds later than the conventional method. However, the 


\section{Journal of Advanced Simulation in Science and Engineering}

disaster detection rate becomes $40 \%$ higher than the conventional one. These experiments were conducted in an actual classroom. Examinees were hard to run because passages in the classroom were narrow and the exit was concentrated in one place. In other words, it was the situation that accumulation of the number of terminals of the running state was not increased quickly. However, the proposed method shows a large value when performing the different behaviors in daily life. Therefore, it shows that the accuracy of the disaster detection of the proposed method is higher than that of the conventional method.

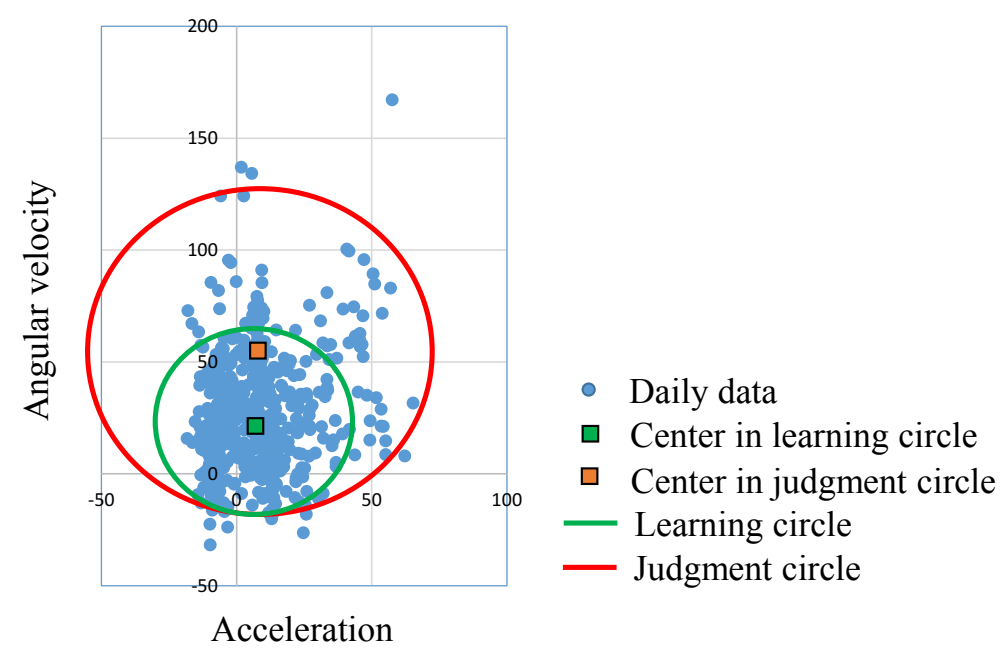

Figure 10: Judgment circle in $5^{\text {th }}$ experiment

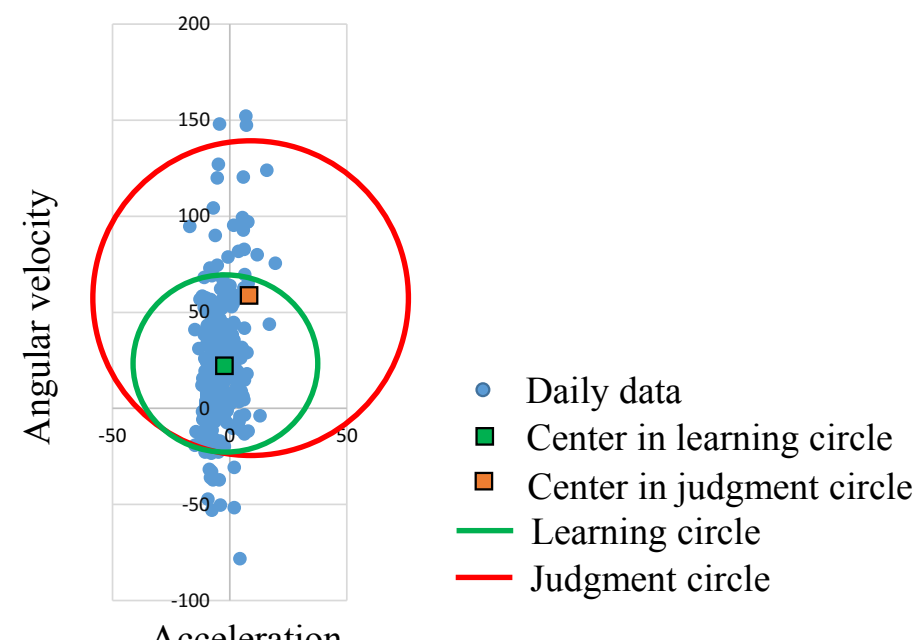

Figure 11: Judgment circle in $7^{\text {th }}$ experiment 
Table 4: Detection time and detection rate

\begin{tabular}{|c|c|c|}
\hline Number of times & $\begin{array}{c}\text { Conventional } \\
\text { method }\end{array}$ & $\begin{array}{c}\text { Proposed } \\
\text { method }\end{array}$ \\
\hline 1 & 12 & 12 \\
\hline 2 & Non-detection & Non-detection \\
\hline 3 & 13 & 13 \\
\hline 4 & Non-detection & 5 \\
\hline 5 & 11 & 12 \\
\hline 6 & Non-detection & Non-detection \\
\hline 7 & 9 & 19 \\
\hline 8 & Non-detection & 5 \\
\hline 9 & Non-detection & 18 \\
\hline 10 & Non-detection & 15 \\
\hline 11 & Non-detection & 14 \\
\hline 12 & Non-detection & $14[\mathrm{~s}]$ \\
\hline 13 & 15 & $77 \%(11 / 13)$ \\
\hline Average detection time & $12[\mathrm{~s}]$ & \\
\hline Disaster detection rate & $38 \%(5 / 13)$ & \\
\hline
\end{tabular}

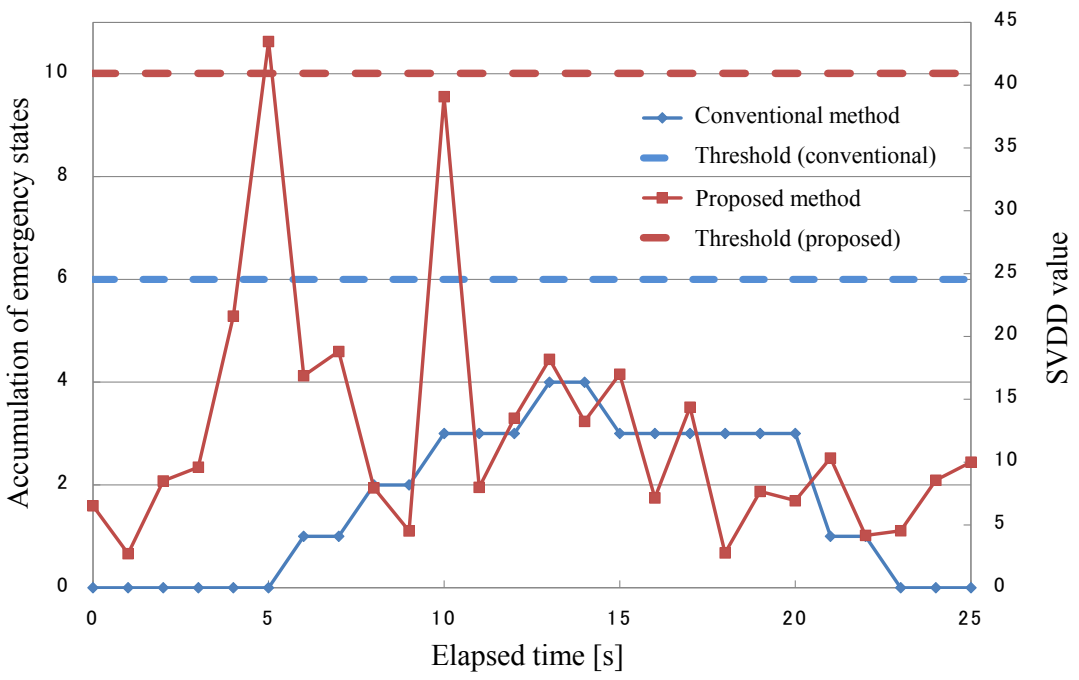

Figure 12: Accumulation of emergency states and SVDD 


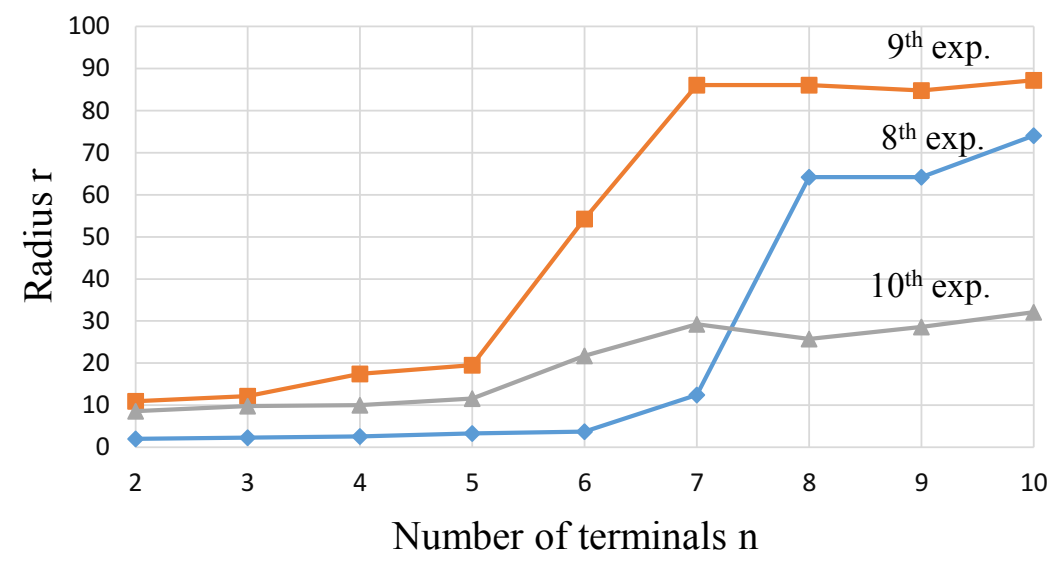

Figure 13: Radius $r$ against the number of terminals $n$

Fig. 13 shows the radius $r$ of judgment circle against the number of terminals $n$ in some experiments. We find that the radius $r$ is increasing according to the increase of the number of terminals $\mathrm{n}$. This is because the radius $\mathrm{r}$ depends on the number of data from terminals. When the number of terminals are more than seven, $r$ comes close to a constant value in each experiment. So we consider at least eight terminals are necessary for disaster detection.

\section{Conclusions}

A lot of people have lost their lives due to sudden disasters such as fire and terrorism. Several evacuation support systems have studied to reduce the number of victims. However these systems have some problems that they have high cost and cannot work correctly in disaster because we have to set many sensors on infrastructure. We have developed the advanced evacuation support system based on MANET named ERESS (Emergency Rescue Evacuation Support System) to solve the above problems.

ERESS is an innovative system that can detect disasters and support evacuation by using only mobile terminals. In this paper, we have proposed a new effective disaster detection method using SVDD. In order to show the effectiveness of the proposed method, we evaluated the detection precision and the detection time of disasters by disaster simulation experiments. As experimental results, we have obtained the followings.

(1) The proposed method can judge the difference in urgent degree.

(2) The proposed method has the time of disaster detection that is equal to the conventional method, and has the detective precision about $40 \%$ higher than the conventional method.

(3) By using the proposed method, we can detect disasters without the influence of the congestion of people.

\section{Acknowledgement}

This research is partially supported by the Grant-in-Aid for Scientific Research (A) (No. 
26240012) from the Ministry of Education, Science, Sport and Culture of Japan, and Research Group Support Grant from Kansai University. The authors thank Kazuya Mori of visiting researcher in Kansai University for his help.

\section{References}

[1] M. Barnes, H. Leather, and D.K. Arvind: Emergency evacuation using wireless sensor networks, in 32nd IEEE Conference on Local Computer Networks (LCN 2007), Dublin, Island of Ireland, 2007, 851-857.

[2] W.H. van Willigen, R.M. Neef, A. van Lieburg, and Martijn C. Schut: WILLEM: a Wireless InteLLigent Evacuation Method, in 2009 Third International Conference on Sensor Technologies and Applications, Athens, Glyfada, 2009, 382-387.

[3] KDDI Corpolation, "DisasterNavi," KDDI, http://www.au.kddi.com/saigaiji_navi/index.html, June 25,2014.

[4] T. Tsunemine, E. Kadokawa, Y. Ueda, J. Fukumoto, T. Wada, K. Ohtsuki, and H. Okada: Emergency Urgent Communications for Searching Evacuation Route in a Local Disaster, in IEEE Consumer Communications and Networking Conference 2008 (CCNC2008), Los Vegas, NV, 2008, 1196-1200.

[5] A. Yamane, Y. Hayakawa, K. Mori, T. Wada, K. Ohtsuki, and H. Okada: Development of Emergency Rescue Urgent Communications in Panic-type Disasters: Immediate Emergency Detections by Evacuee's Behavior, in JSST Multi Dimension Mobile Communication Network Society 2010 (No.JSST-MDMC2010-2-04), Miyagi, 2010. (in Japanese)

[6] K. Mori, A. Yamane, Y. Hayakawa T. Wada, K. Ohtsuki, and H. Okada: Development of Emergency Rescue Evacuation Support System (ERESS) in Panic-type Disasters: Immediate Emergency Detections by Machine Learning, in International Technical Conference on Circuits/Systems, Computers and Communications (ITC-CSCC), Gyeongju, Korea, 2011, 69-72.

[7] K. Mori, A. Yamane, Y. Hayakawa, T. Wada, K. Ohtsuki, and H. Okada: Development of Emergency Rescue Evacuation Support System (ERESS) in Panic-type Disasters: Disaster Recognition Algorithm by Support Vector Machine, IEICE Trans. on Fundamentals, E96-A:2(2013), 649-657.

[8] H. Higuchi, J. Fujimura, T. Nakamura, K. Kogo, K. Tsudaka, T. Wada, H. Okada, and K. Ohtsuki: Disaster Detection by Statistics and SVM for Emergency Rescue Evacuation Support System, in 43rd Internet Conference on Parallel Processing Workshops (IC$C P W)$, Minneapolis, MN, 2014, 349-354.

[9] H. Murotsu, M. Fujimoto, T. Suzuki, H. Ebara, T. Wada, H. Okada: A Novel Evacuation Route Search Algorithm for Route Distribution of Evacuees Groups in Fire Disasters, in 2014 Australasian Telecomunication Networks and Applications Conference (ATNAC), Southbank, VIC, 2014, 182-188.

[10] Y. Jeong, I. Kang, M. Jeong, Senior Member, IEEE, and D. Kong: A New Feature Se 
Journal of Advanced Simulation in Science and Engineering

lection Method for One-Class Classification Problems, IEEE Trans. on Systems, Man, and Cybemetics, Part C: Applications and Reviews, 42:6(2012), 1500-1509. 